\title{
Bladder Preservation with Neoadjuvant Chemotherapy Followed by Concurrent Chemoradiation for the Treatment of Muscle-invasive Carcinoma of the Bladder: A Single-Center Experience
}

\author{
Chinmayee Agrawal ${ }^{1}$ Saurabh Bansal ${ }^{1}$ Manoj Biswas ${ }^{2} \quad$ Meenu Gupta $^{1} \quad$ Vipul Nautiyal ${ }^{1}$ \\ Mushtaq Ahmed ${ }^{1}$ \\ ${ }^{1}$ Department of Radiation Oncology, Cancer Research Institute, \\ Address for correspondence Dr. Saurabh Bansal, MD, \\ SRHU, Dehradun, Uttarakhand, India \\ ${ }^{2}$ Department of General Surgery, HIMS, SRHU, Dehradun, \\ Uttarakhand, India \begin{abstract}
Department of Radiation Oncology, Cancer Research Institute, SRHU, Dehradun 248016, Uttarakhand, India (e-mail: drbansal1985@gmail.com).
\end{abstract}

\section{South Asian J Cancer 2020;9:121-125.}

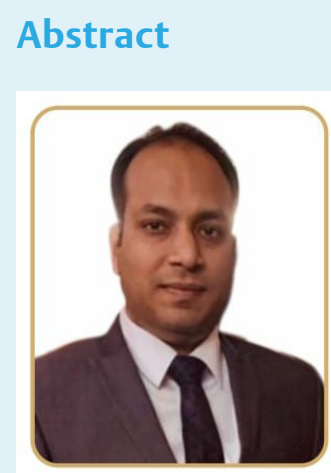

Dr. Saurabh Bansal, MD

Keywords

- bladder preservation

- muscle-invasive

bladder cancer

- neoadjuvant

chemotherapy
Purpose The purpose of the study was to evaluate the short-term response and acute toxicities in muscle-invasive carcinoma urinary bladder treated with neoadjuvant chemotherapy followed by concurrent chemoradiation.

Materials and Methods Thirty patients with muscle-invasive bladder cancer were treated with three cycles of neoadjuvant chemotherapy every 3 weeks. Response assessment was done after 4 weeks with repeat cystoscopy and imaging. Responders were treated with concurrent chemoradiation $60 \mathrm{~Gy} / 30 \#$ at $2 \mathrm{~Gy} / \#$ along with weekly injection cisplatin $35 \mathrm{mg} / \mathrm{m}^{2}$. Response assessment was done by new response evaluation criteria in solid tumors (version 1.1). Treatment-related acute toxicities were scored using common terminology criteria for adverse events version 4.0.

Results Of the 30 patients, 25 patients responded to neoadjuvant chemotherapy with complete response in 17 patients (56.67\%) and partial response in eight patients (26.66\%). Five patients (16.66\%) showed poor response and were advised radical cystectomy, of which four underwent radical cystectomy and one patient opted for concurrent chemoradiation. Of 26 patients who completed chemoradiation, complete response was seen in 21 patients (80.76\%) and partial response was seen in four patients (15.38\%). Only one patient developed progression of disease in the form of lung metastasis. All the patients with residual disease were advised to undergo salvage cystectomy. Among the patients receiving chemoradiation, grade 2 cystitis and diarrhea was seen in 10 patients (38.46\%) and four patients (15.38\%), respectively. Only one patient developed grade 3 diarrhea.

Conclusion Bladder preservation treatment is an effective, safe, and convenient option for patients presenting with muscle-invasive carcinoma bladder. Neoadjuvant chemotherapy followed by chemoradiation was well-tolerated with an acceptable rate of complications.
How to cite this article: Agrawal C, Bansal S, Biswas M, Gupta M, Nautiyal V, Ahmed M. Bladder Preservation with Neoadjuvant Chemotherapy Followed by Concurrent Chemoradiation for the Treatment of Muscle-invasive Carcinoma of the Bladder: A SingleCenter Experience. South Asian J Cancer 2020;9(3):121-125.

DOI https://doi.org/10.1055/s-0041-1723076 ISSN 2278-330X.
(C) 2020. MedIntel Services Pvt Ltd.

This is an open access article published by Thieme under the terms of the Creative Commons Attribution-NonDerivative-NonCommercial-License, permitting copying and reproduction so long as the original work is given appropriate credit. Contents may not be used for commercial purposes, or adapted, remixed, transformed or built upon. (https://creativecommons.org/licenses/by-nc-nd/4.0/)

Thieme Medical and Scientific Publishers Pvt. Ltd., A-12, 2nd Floor, Sector 2, Noida-201301 UP, India 


\section{Introduction}

Carcinoma bladder is the ninth most common carcinoma worldwide, estimating approximately $2 \%$ of all the cancer death. ${ }^{1}$ It is a common malignancy of increasing age group, with a median age of 68 years at the time of diagnosis. ${ }^{2}$ The main contributor to bladder carcinoma is smoking, with smokers running a triple risk. The most common histology is transitional cell carcinoma (TCC), accounting for over $90 \%$ cases. ${ }^{3}$ Hematuria and burning micturition are the most common presenting complaints seen in $>80 \%$ of the patients. ${ }^{4}$

The gold standard management of muscle-invasive carcinoma bladder remains the radical cystectomy with bilateral pelvic lymph node dissection which is associated with recurrence-free survival of $68 \%$ at 5 years and $66 \%$ at 10 years. ${ }^{5}$ The early- and late-occurring morbidities include risk of infection, urinary leak, hemorrhage, and pelvic lymphoceles. The construction of neobladder cannot substitute for a person's original bladder and is associated with acute and long-term neuromechanical and metabolic complications. ${ }^{6}$

Various studies have shown that bladder preservation therapies offer results comparable to radical cystectomy, with the additional advantage of retaining a functional bladder. ${ }^{7}$ The bladder preservation therapies incorporate maximal transurethral resection of tumor, followed by concurrent chemoradiation. ${ }^{8}$

There has been an emerging role of neoadjuvant chemotherapy due to high propensity for distant failure in carcinoma bladder. There has been a survival benefit of approximately $5 \%$ at 5 years for neoadjuvant cisplatin-based combination chemotherapy. ${ }^{9}$ Complete response to induction treatment helps to identify persons who may be specifically suitable for bladder sparing, while patients with poor response to induction therapy can directly proceed to radical cystectomy. ${ }^{7}$

Selective bladder preservation treatment is a reasonable alternative approach in patients presenting with muscle-invasive carcinoma bladder. ${ }^{10}$ It has been developed to improve quality of life (QOL) of patients who want to maintain urinary function without a diversion and are not candidates for surgery. ${ }^{11}$ Thus, the aim of our study is to evaluate short-term outcomes of bladder preservation in muscle-invasive carcinoma bladder after neoadjuvant chemotherapy followed by concurrent chemoradiation.

\section{Materials and Methods}

\section{Patient Selection}

This prospective study was conducted at the Department of Radiation Oncology, Cancer Research Institute, SRHU University, over a period of 12 months. Thirty patients with a primary diagnosis of carcinoma urinary bladder with the Eastern Cooperative Oncology Group (ECOG) performance status $\leq 2$ were recruited after obtaining written informed consent and ethical clearance certificate from institutional ethics committee. All patients were staged according to the American Joint Committee on Cancer 8th edition TNM Staging. Exclusion criteria included informed consent not granted, any other malignancy, evidence of distant metastasis, morbid conditions, psychiatric illness, and patients with tissue diagnosis other than TCC.

\section{Treatment}

After the baseline assessment, the patients were treated with three cycles of neoadjuvant chemotherapy with injection gemcitabine $\left(1000 \mathrm{mg} / \mathrm{m}^{2}\right)$ on days 1 and 8 and injection cisplatin $\left(75 \mathrm{mg} / \mathrm{m}^{2}\right)$ on day 1 every 3 weeks. Response assessment was done after 4 weeks of the last neoadjuvant chemotherapy with repeat cystoscopy and imaging. Responders were treated with concurrent chemoradiation $60 \mathrm{~Gy} / 30 \#$ at $2 \mathrm{~Gy} / \# 5$ days a week over 6 weeks along with weekly cisplatin $35 \mathrm{mg} / \mathrm{m}^{2}$ injection.

All patients underwent computed tomography (CT) simulation, and three-dimensional conformal planning was performed. Radiotherapy was given with the help of shrinking field technique. The first phase included $40 \mathrm{~Gy}$ with fraction size $2 \mathrm{~Gy}$ in 20 fractions to the whole pelvis, encompassing the entire bladder and pelvic lymph nodes. In the second phase, field was reduced to cover the gross tumor volume with $2 \mathrm{~cm}$ margin, and the dose given was $20 \mathrm{~Gy}$ in 10 fractions to a total dose of $60 \mathrm{~Gy}$. Complete blood counts and serum creatinine levels were checked every week.

All patients were evaluated 6 weeks after completion of therapy with cystoscopy, contrast-enhanced CT abdomen/ pelvis, and chest X-ray. Assessment of response to the treatment was done using the new response evaluation criteria in solid tumors: revised RECIST guidelines version 1.1. Treatment-related acute toxicities in the form of hematology (anemia, neutropenia, and thrombocytopenia) and nonhematology (vomiting, diarrhea, and cystitis) were scored using common terminology criteria for adverse events version 4.0.

\section{Data Management and Statistical Analysis}

Interpretation and analysis of obtained results were performed using software SPSS version 22 by the application of descriptive methods (mean, rate, ratio, proportional, etc.). Categorical variables were expressed as frequencies and percentages.

\section{Results}

Maximum patients were in the seventh decade (median age of 61.4 years), with a male:female ratio of $14: 1$. The most common presenting symptoms were hematuria and burning micturition seen in $73.33 \%$ of the patients. Majority of the patients had T2b disease, accounting for 18 patients (60.0\%).

Of the 30 patients, 26 patients completed planned three cycles of neoadjuvant chemotherapy. Three patients received only two courses of chemotherapy because of grade 3 neutropenia and thrombocytopenia, resulting in a prolonged recovery. Only one patient received one cycle of chemotherapy due to grade 3 gastrointestinal (GI) side effects. 
Twenty-five patients responded well to neoadjuvant chemotherapy. Complete response was seen in 17 patients (56.67\%) and partial response was seen in eight patients (26.66\%). Five patients had a poor response and were advised radical cystectomy, of which four underwent radical cystectomy and one patient opted for concurrent chemoradiation.

Hematological toxicities in the form of grade 2 anemia, thrombocytopenia, and neutropenia were seen in 3.33\% (one patient), 10\% (three patient) and 13.33\% (four patients), respectively. Grade 3 neutropenia was seen in 10\% (three patients) patients, and all of them received growth colony-stimulating factors. Among nonhematological toxicities, grade 1 nausea was seen in four patients (13.33\%). Grade 2 vomiting and diarrhea was seen in three patients (10\%) and one patient, respectively.

Of the 26 patients who completed chemoradiation, complete response was seen in 21 patients (80.76\%), partial response was seen in four patients (15.38\%), and one patient developed progression of disease in the form of lung metastasis. All the patients with the residual disease were advised to undergo salvage cystectomy. Two patients registered for salvage cystectomy and one patient for palliative chemotherapy, owing to developing lung metastasis. Two patients did not opt for any further treatment.

Among patients receiving chemoradiation, the most common toxicity was cystitis followed by diarrhea. Grade 1 and 2 cystitis was seen in four patients (15.38\%) and 10 patients (38.46\%), respectively. Grade 1 and 2 diarrhea occurred in six patients (23.07\%) and four patients (15.38\%), respectively. Only one patient developed grade 3 diarrhea.

\section{Discussion}

The gold standard treatment of muscle-invasive bladder cancer is radical cystectomy. However, the misfortune of losing one's own bladder function is a major kind of mutilation, resulting in anxiety and poor QOL. Thus, there has been a change in treatment paradigm shifting from radical cystectomy to bladder preservation approach.

Different studies have shown bladder preservation therapy as an effective, safe, and convenient approach with acceptable toxicities when compared with gold standard radical cystectomy along with a comparable disease-free and overall survival. Bladder preserving treatment has helped majority of patients to maintain good QOL, to retain good bladder function, and to preserve sexual function without compromising long-term survival. Trimodality bladder preservation approach includes transurethral resection of a bladder tumor (TURBT), followed by concurrent chemoradiation with or without neoadjuvant chemotherapy, which is becoming a promising treatment in bladder cancer.

During follow-up, a significant number of patients of bladder cancer presented with distant failure; hence, there is a need to intensify the systemic treatment. This has led to the emergence of neoadjuvant chemotherapy in the treatment, which can decrease the incidence of distant metastasis. A significant survival benefit in association with platinum-based chemotherapy was found, which was equivalent to $5 \%$ improvement in survival at 5 years. ${ }^{12}$

The present study aimed to study the efficacy and tolerance of neoadjuvant chemotherapy, followed by concurrent chemoradiation in muscle-invasive bladder cancer. A total of 30 patients were enrolled in the study and were treated with three cycles of neoadjuvant chemotherapy followed by concurrent chemoradiation.

The baseline characteristics in the present study were consistent and comparable with the literature. In the study done, $30 \%$ (nine patients) of the patient population were in the seventh decade of life, with a median age of 61.4 years, which is comparable with the study done by Hafeez et $\mathrm{al}^{7}$ and Perdonà et $\mathrm{al},{ }^{13}$ in which the median age was 65 and 63 years, respectively. In the present study, $93.33 \%$ patients were male (male:female ratio: 14:1). In a pivotal study conducted by Hafeez et al, ${ }^{7}$ the patients were predominantly male (87.23\%). Similarly, male:female ratio was $7.3: 1$ in a study done by Mitin et al. ${ }^{14}$

Majority of the patients presented with T2b (60\%) and T3 (26.6\%) disease in our study, which was similar to the study done by Tunio et al, ${ }^{15}$ and in which $31.2 \%$ of patients presented with T2 and $46.9 \%$ with T3 disease. Similarly, 77\% and $19 \%$ of patients presented with $\mathrm{T} 2$ and $\mathrm{T} 3$ disease in a study done by Sabaa et $\mathrm{a}^{16}$ In our study, all the patients had an ECOG performance score of 0 to 2, which was comparable to the studies done by Mitin et $\mathrm{al}^{14}$ and Perdonà et $\mathrm{al}^{13}$ The most common presenting symptom were hematuria and burning micturition seen in $73.33 \%$ (22) of patients.

Of the 30 patients, 26 patients completed planned three cycles of neoadjuvant chemotherapy. Three patients received only two cycles, and one patient received one cycle of chemotherapy because of treatment-related toxicity. In the study done by Tunio et al, ${ }^{15}$ two patients (4.6\%) did not receive planned courses of neoadjuvant chemotherapy due to grade 4 neutropenia and thrombocytopenia. In a study done by Sadzikowska et al, ${ }^{17}$ only $78 \%$ of patients completed planned neoadjuvant chemotherapy cycles, while $22 \%$ of patients did not receive planned chemotherapy cycles.

In the present study, four patients (13.3\%) received gemcitabine-carboplatin-based chemotherapy instead of gemcitabine-cisplatin due to raised serum creatinine. Similar deviation from standard cisplatin-based chemotherapy was seen in the study done by Hafeez et al, ${ }^{7}$ in which 12 patients (12.7\%) received gemcitabine and carboplatin.

Twenty-five patients demonstrated a favorable response to neoadjuvant chemotherapy, while five patients showed a poor response. Among responders, complete response was seen in 17 patients (56.6\%) and partial response in eight patients (26.67\%). Jadwiga et $\mathrm{al}^{17}$ in their study demonstrated complete response in eight patients (30\%), partial response in 13 patients (48\%), and stable disease in six patients (22\%). In a pioneer study, conducted by Hafeez et $\mathrm{al}^{7}{ }^{7}$ $88 \%$ of patients illustrated a good response, with $60 \%$ of patients achieving complete response following neoadjuvant chemotherapy. In a study done by Tunio et al, ${ }^{15} 78 \%$ of patients showed complete response to neoadjuvant chemotherapy. 
Hematological toxicity attributable to neoadjuvant chemotherapy included grade 3 neutropenia seen in three patients (10\%). Grade 2 anemia occurred in $3.33 \%$ and grade 2 thrombocytopenia in $10 \%$ of patients. Nonhematological toxicity included vomiting and diarrhea with 10\% and 3\% presenting with grade 2 toxicities, respectively. In a study done by Tunio et al, ${ }^{15}$ grade 3 neutropenia and thrombocytopenia was seen in $14.6 \%$ and $4.9 \%$ patients, respectively. Grade 3 nausea and vomiting was seen in $17.1 \%$ and anorexia in $7.1 \%$ of patients, respectively. In a study conducted by Sadzikowska et al, ${ }^{17} 22 \%$ and $4 \%$ of patients presented with grade 3 or grade 4 neutropenia and thrombocytopenia, respectively.

All the 26 patients were able to complete the entire course of radiation. Majority of patients (73\%) received 3 to 5 cycles of concurrent chemotherapy, while only 19.2\% (five patients) received $<3$ cycles of concurrent chemotherapy. Two patients (7.6\%) did not receive any concurrent chemotherapy. Sadzikowska et al ${ }^{17}$ in their study were able to give concurrent chemotherapy to $33 \%$ of the patients, while it was omitted for one course in six patients in a study done by Tunio et $\mathrm{a}^{15}$

Postchemoradiation, complete response and partial response were seen in 21 patients (80.7\%) and four patients (15.3\%), respectively. Only one patient developed progression of disease. All the patients with residual disease were advised salvage cystectomy, while one patient with lung metastasis was planned for palliative chemotherapy. In a study conducted by Tunio et al, ${ }^{15}$ complete response was demonstrated in 32 patients (78.04\%). Of nine patients (21.95\%), who did not achieve complete response, six underwent salvage cystectomy and three were treated with salvage chemotherapy. Sadzikowska et al, ${ }^{17}$ in their pioneering study, showed complete response in 18 patients (67\%), partial response in two patients (8\%), and stable disease in seven patients (25\%).

Among patients receiving concurrent chemoradiation, most common toxicity was cystitis followed by diarrhea. Grade 2 cystitis was seen in 10 patients (38.46\%), while grade 2 diarrhea occurred in four patients (15.38\%). Sabaa et al ${ }^{16}$ recorded grade 1 or 2 radiation cystitis in $51.9 \%$ of patients and grade 1 or 2 diarrhea in $35.6 \%$ of patients. Similarly, Tunio et $\mathrm{al}^{15}$ illustrated grade 3 diarrhea in $18.7 \%$ of patients and cystitis in $18.7 \%$ of patients.

Given these findings, we believe that bladder preservation treatment with neoadjuvant chemotherapy followed by chemoradiation is well-tolerated with an acceptable rate of complications and should be recommended in patients who refuse surgery or are not candidates for surgery.

\section{Conclusion}

Our results support that bladder preservation treatment is an effective, safe, and convenient option for patients presenting with muscle-invasive carcinoma bladder. Although the sample size was small and follow-up period was shorter, our study has shown encouraging results with bladder preservation treatment, a high probability of local response, and acceptable toxicity in properly selected patients.
Financial Support and Sponsorship

Nil.

\section{Conflicts of Interest}

None declared.

\section{Acknowledgment}

Authors are highly thankful to the SRH University for permitting this research study and Uttarakhand State Council for science and technology assistance in data management and compiling.

\section{References}

1 Rene NJ, Cury FB, Souhami L. Conservative treatment of invasive bladder cancer. Curr Oncol 2009;16(4):36-47

2 Balar A, Bajorin DF, Milowsky MI. Management of invasive bladder cancer in patients who are not candidates for or decline cystectomy. Ther Adv Urol 2011;3(3):107-117

3 Zhang S, Yu YH, Zhang Y, Qu W, Li J. Radiotherapy in muscle-invasive bladder cancer: the latest research progress and clinical application. Am J Cancer Res 2015;5(2):854-868

4 Bellmunt J, Orsola A, Leow JJ, Wiegel T, De Santis M, Horwich A; ESMO Guidelines Working Group. Bladder cancer: ESMO Practice Guidelines for diagnosis, treatment and follow-up. Ann Oncol 2014;25(Suppl 3) :iii40-iii48

5 Stein JP, Lieskovsky G, Cote R, et al. Radical cystectomy in the treatment of invasive bladder cancer: long-term results in 1,054 patients. J Clin Oncol 2001;19(3):666-675

6 Beena K, Nambiar VR, Dinesh M. Tri-modality treatment in muscle invasive bladder cancer - What is the current status. Amrita J Med 2013;9:14-21

7 Hafeez S, Horwich A, Omar O, et al. Selective organ preservation with neo-adjuvant chemotherapy for the treatment of muscle invasive transitional cell carcinoma of the bladder. $\mathrm{Br}$ J Cancer 2015;112(10):1626-1635

8 Tunio MA, Asiri MA, Fareed M, et al. Bladder preservation with concurrent radiotherapy and gemcitabine following maximal transurethral resection for muscle invasive bladder cancer: single institutional experience. J Nucl Med Radiat Ther 2012;3:121-128

9 Grossman HB, Natale RB, Tangen CM, et al. Neoadjuvant chemotherapy plus cystectomy compared with cystectomy alone for locally advanced bladder cancer. N Engl J Med 2003;349(9):859-866

10 Sternberg CN, Yagoda A, Scher HI, et al. Preliminary results of M-VAC (methotrexate, vinblastine, doxorubicin and cisplatin) for transitional cell carcinoma of the urothelium. J Urol 1985;133(3):403-407

11 FernandoSA,SandlerHM.Organpreservationinmuscle-invasive bladder cancer. Oncology (Williston Park) 2005;19(3): 334-339, discussion 339-340, 345, 349, 350-353

12 Advanced Bladder Cancer (ABC) Meta-analysis Collaboration. Neoadjuvant chemotherapy in invasive bladder cancer: update of a systematic review and meta-analysis of individual patient data advanced bladder cancer (ABC) meta-analysis collaboration. Eur Urol 2005;48(2):202-205, discussion 205-206

13 Perdonà S, Autorino R, Damiano R, et al. Bladder-sparing, combined-modality approach for muscle-invasive bladder cancer: a multi-institutional, long-term experience. Cancer 2008; 112(1):75-83

14 Mitin T, George A, Zietman AL, et al. Long-term outcomes among patients who achieve complete or near-complete responses after the induction phase of bladder-preserving combined-modality therapy for muscle-invasive bladder cancer: A pooled analysis of NRG oncology/RTOG 9906 and 0233. Int J Radiat Oncol Biol Phys 2016;94(1):67-74 
15 Tunio MA, Hashmi A, Rafi M, Qayyum A, Masood R. Bladder preservation by neoadjuvant chemotherapy followed by concurrent chemoradiation for muscle-invasive bladder cancer: experience at Sindh Institute of Urology \& Transplantation (SIUT). J Pak Med Assoc 2011;61(1):6-10

16 Sabaa MA, El-Gamal OM, Abo-Elenen M, Khanam A. Combined modality treatment with bladder preservation for muscle invasive bladder cancer. Urol Oncol 2010;28(1):14-20
17 Sadzikowska JN, Kowalska T, Jakubowicz J, Szpytma T. Conservative treatment in patients with muscle-invasive bladder cancer by transurethral resection, neoadjuvant chemotherapy with gemcitabine and cisplatin, and accelerated radiotherapy with concomitant boost plus concurrent cisplatin assessment of response and toxicity. Rep Pract Oncol Radiother 2008;13:300-308 